\title{
Numerical Modeling the Effects of Chamfer and Hone Cutting Edge Geometries on Burr Formation
}

\author{
Muhammad Asad $^{\mathrm{a}} \uparrow$, Faramarz Djavanroodi ${ }^{\mathrm{b}}$, Hassan Ijaz ${ }^{\mathrm{c}}$, Muhammad Azhar Ali Khan ${ }^{\mathrm{d}}$, Muhammad \\ Usman Rashid $^{\mathrm{e}}$, Tarek Mabrouki ${ }^{\mathrm{f}}$ \\ a, b, dMechanical Engineering Department, Prince Mohammad Bin Fahd University, Al-Khobar, \\ SAUDI ARABIA \\ ${ }^{\mathrm{c}}$ Mechanical and Materials Engineering Department, University of Jeddah, Jeddah, SAUDI ARABIA \\ ${ }^{\mathrm{e}}$ Civil Engineering Department, University of Management and Technology, Lahore, PAKISTAN \\ ${ }^{\mathrm{f}}$ Applied Mechanics and Engineering Laboratory, University of Tunis El Manar, ENIT, Tunis, \\ TUNISIA
}

\begin{abstract}
A finite element based numerical model to simulate orthogonal machining process and associated burr formation process has been developed in the presented work. To incorporate simultaneous effects of mechanical and thermal loadings in high speed machining processes, Johnson and Cook's thermo-visco-plastic flow stress model has been adopted in the conceived numerical model. A coupled damage-fracture energy approach has been used to observe damage evolution in workpiece and to serve as chip separation criterion. Simulation results concerning chip morphology, nodal temperatures, cutting forces and end (exit) burr have been recorded. Model has been validated by comparing chip morphology and cutting force results with experimental findings in the published literature. Effects of cutting edge geometries [Hone and Chamfer (T-land)] on burr formation have been investigated thoroughly and discussed in length. To propose optimum tool edge geometries for reduced burr formation in machining of an aerospace grade aluminum alloy AA2024, numerical analyses considering multiple combinations of cutting speed (two variations), feed (two variations) and tool edge geometries [Hone edge (two variations), Chamfer edge (four variations)] have been performed. For chamfer cutting edge, the "chamfer length" has been identified as the most influential macro geometrical parameter in enhancing the burr formation. Conversely, "chamfer angle" variation has been found least effecting the burr generation phenomenon.
\end{abstract}

Key-Words: - Finite element analysis, machining simulation, cutting edge geometry, burr Received: February 10, 2020. Revised: September 13, 2020. Accepted: October 1, 2020. Published: October 7, 2020.

\section{Introduction}

Aluminum alloys are amongst the popular metallic materials commonly used in automotive and aeronautical industries due to their attractive weightto-strength ratio properties. While, their high machinability index (300-1500) allows them to be dry machined [1]. This makes them ideal materials for green manufacturing. Nevertheless, dry machining of aluminum alloys may result in augmented burr formation on workpiece edges [2-3]. Burr, the unwanted sharp material accumulates on edges of machined work parts, as cutting tool exit the workpiece or machining is performed near workpiece edges. Removal of burr from machined part edges is a must process to be carried out using various methods [4], before further processing on machined parts or their use in an assembly environment. The non-value added deburring process involves wastage of resources of energy, money and time. Literature study shows that burr formation can be adequately reduced to minimum levels employing appropriate selection of tool geometry and cutting angles $[3,5]$, altering the workpiece geometry [4], optimum cutting parameters selection $[2,4,6]$ and using MQL and cryogenic assisted machining [7-8] techniques. In the present work a finite element (FE)-based approach to simulate and predict burr formation in 
dry machining of an aluminum alloy AA2024 has been proposed. While, FE-methods have been successfully used to simulate multiphysical design problems for last few decades. Machining simulations using chamfer (T-land) and hone tool cutting edge geometries have been performed. Relative effects of macro geometry parameters of chamfer edge tools including chamfer length $\left(\ell_{\beta}\right)$ and chamfer angle $\left(\gamma_{\beta}\right)$ on cutting force variations, chip morphology evolution and exit (end) burr formation have been discussed. Effects of micro geometry parameters have been ignored. Later, geometry parameters effects must be considered in micro machining scenarios, when tool edge dimensions are equal or larger than feed values [9]. Objective is to propose an optimum tool edge geometry (hone and chamfer) to reduce burr formation when dry machining AA2024 with multiple cutting parameters combinations of cutting speed and feed values (for semi-finish to finish cut cases).

For comprehension of burr phenomenon, a numerical model based on 2D FE approach for fundamental orthogonal machining case has been proposed. Simulation results of chip morphology, cutting forces and burr are compared with existing experimental literature $[2,6,10]$.

\section{Machining model geometry, material behavior and hypothesis}

The tool and workpiece geometry is modeled in a commercial FEA software Abaqus ${ }^{\circledR}$. The conceived model is shown in Fig.1. Three distinct parts of workpiece: machined workpiece, chip separation zone and feed (uncut chip) are modeled separately and onwards attached together using tie-constraint algorithm (Abaqus ${ }^{\circledR}$ inbuilt feature), such that workpiece behave as single entity during cutting simulation. Separate modeling of various parts of workpiece allows to define different material behavior properties (defined later in this section) in different sections of workpiece and ease in defining interaction properties.

Fully constrained boundary conditions are defined only on lower surface of workpiece, allowing material flow and deformation of workpiece elements elsewhere. Whereas, tool can move in negative $\mathrm{x}$-axis direction with the defined cutting speed. Six different tool cutting edge geometries: two hone edge variations and four chamfer edge variations are considered in this work, as shown in Fig.1. Tool angles (positive rake angle $=17.5^{\circ}$ and relief angle $=$ $\left.7^{\circ}\right)$ and hone edge radius $(\mathrm{r} \beta=20 \mu \mathrm{m})$ are similar to those used in the experimental work [10]. While, rest of five tool edge geometries: one hone edge $(\mathrm{r} \beta=$
$5 \mu \mathrm{m}$ ) and four chamfer edge (with chamfer length $(1 \beta)=0.1 \mathrm{~mm}$ and $0.2 \mathrm{~mm}$ and chamfer angle $(\gamma \beta)=$ $15^{\circ}$ and $25^{\circ}$ ) are modeled here to propose optimum tool edge geometry to reduce the burr formation. In Fig. 1, various chamfer edge geometries are represented with their equivalent hone radius (req). As overall simulation results using chamfer edges would be similar if equivalent hone edge radius would have been used (explained later in next section).

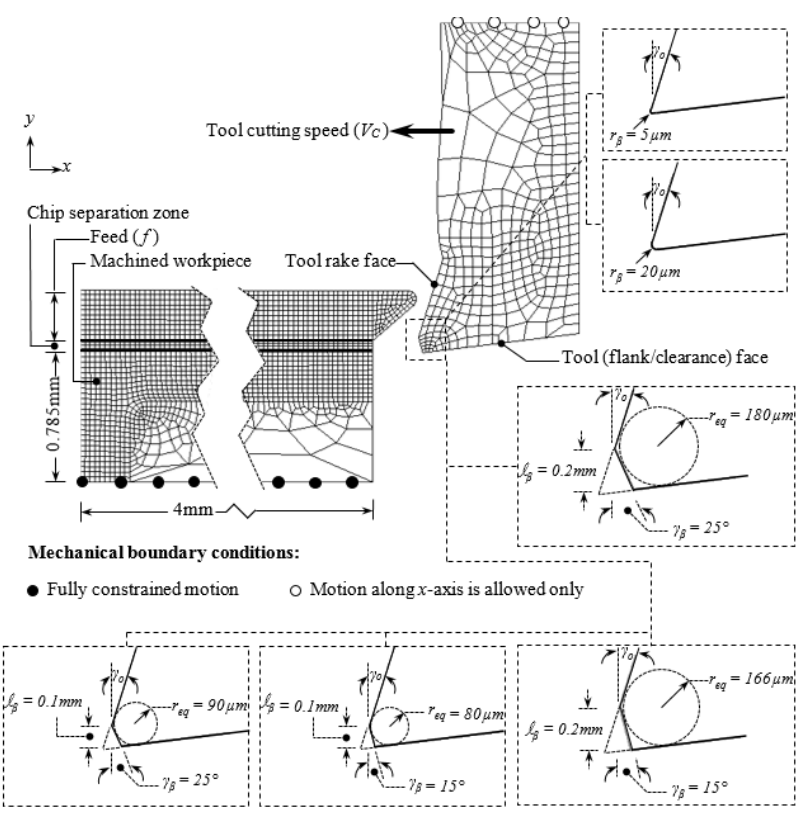

Fig. 1. Two dimensional workpiece and tool machining model with various tool edge geometries.

Plain strain hypothesis has been adopted here to perform 2D orthogonal machining simulation. Similar approach has also been used previously in published literature [11-13]. Plain strain continuum elements, CPE4RT, are used to mesh tool and workpiece. These elements allow to record thermomechanical behaviour when coupled temperaturedisplacement analysis are performed. Toolworkpiece interaction is defined using Coulomb friction law. Cutting tool (tungsten carbide insert) is assigned with its elastic properties only. On the other hand, Johnson and Cook's thermos-elasto-visco plastic constitutive model [14] is employed for workpiece to simulate its behavior during machining process (equation 1). To realize chip separation, material damage and fracture energy approaches have been considered [15-16]. Johnson and Cook's shear failure model (equation 2) is used to calculate failure strain. While, equation 3 is used to calculate scalar damage initiation parameter, $\omega$. 


$$
\begin{aligned}
& \bar{\sigma}_{J C}=\underbrace{\left(A+B \bar{\varepsilon}^{n}\right)}_{\text {Elasto plastiderm }} \underbrace{\left[1+C \ln \left(\frac{\dot{\bar{\varepsilon}}}{\overline{\bar{\varepsilon}}_{0}}\right)\right]}_{\text {Viscosityterm }} \underbrace{\left[1-\left(\frac{T-T_{r}}{T_{m}-T_{r}}\right)^{m}\right]}_{\text {Softeningterm }} \\
& \overline{\bar{\varepsilon}}_{0 i}=\left[D_{1}+D_{2} \exp \left(D_{3} \frac{P}{\bar{\sigma}}\right)\right]\left[1+D_{4} \ln \left(\frac{\dot{\bar{\varepsilon}}}{\overline{\bar{\varepsilon}_{0}}}\right)\right]\left[1+D_{5}\left(\frac{T-T_{r}}{T_{m}-T_{r}}\right)\right] \\
& \omega=\sum \frac{\Delta \bar{\varepsilon}}{\bar{\varepsilon}_{0 i}}
\end{aligned}
$$

Now as the damage initiation parameter accumulates to value of "one", the damage evolution (D) is subsequently calculated using equations 4 and 5 . A numeric value of "one" for damage evolution parameter (D) shows that material stiffness is fully degraded in a specific material element. Onwards, that material element or set of elements can be removed from mesh, allowing chip separation from workpiece.

$$
\begin{aligned}
& D=1-\exp \left(-\int_{0}^{\bar{u}} \frac{\bar{\sigma}}{G_{f}} d \bar{u}\right) \\
& D=\frac{\bar{u}}{\bar{u}_{f}}
\end{aligned}
$$

Equation 4, is used in feed area (uncut chip section) and equation 5 , is used to calculate damage evolution is chip-separation-zone. In feed area (uncut chip section), the damage evolution parameter (D) never approaches to "one". This allows chip section elements to remain intact with mesh even acquiring very high value of $D$ (close to one). However, chip section elements having high accumulated value of "D" represent highly deformed set of elements, contributing towards evolution of segmented chip morphology with sharp segments (shown in next section). In equation 4 , Gf represents material fracture energy calculated using equation 6, while, displacement at failure is calculated using equation 7 .

$$
\begin{aligned}
& \left(G_{f}\right)_{I, I I}=\frac{1-v^{2}}{E}\left(K_{C}^{2}\right)_{I, I I} \\
& \bar{u}_{f}=\frac{2 G_{f}}{\sigma_{y}}
\end{aligned}
$$

Furthermore, as high strains ( $>5 \%)$ during the cutting simulation are expected, therefore, to incorporate geometric nonlinearity, "NLGEOM" option in Abaqus/Explicit is activated.
Abaqus/Explicit uses explicit central difference method as nonlinear solution algorithm.

\section{Results and discussions}

Machining simulation for multiple combinations of cutting speed $\left(\mathrm{V}_{\mathrm{C}}=800\right.$ and $\left.400 \mathrm{~m} / \mathrm{min}\right)$, feed $(f=0.3$ and $0.4 \mathrm{~mm} / \mathrm{rev}$ ) and tool edge [hone edge: $\mathrm{R}_{\mathrm{n}}=5$ and $20 \mu \mathrm{m}$, chamfer edge: $\left(\ell_{\beta}=0.1 \mathrm{~mm}, \gamma_{\beta}=15^{\circ}\right),\left(l_{\beta}=\right.$ $\left.0.1 \mathrm{~mm}, \gamma_{\beta}=25^{\circ}\right),\left(\ell_{\beta}=0.2 \mathrm{~mm}, \gamma_{\beta}=15^{\circ}\right)$ and $\left(\ell_{\beta}=\right.$ $\left.0.2 \mathrm{~mm}, \gamma_{\beta}=25^{\circ}\right)$ ] have been carried out. For presentation purpose chamfer edges are represented with their equivalent hone edge radius, as shown in Fig.1. While, in actual simulation work chamfer edges have been used. Numerical results of cutting forces and chip morphology for four different combinations of cutting speed and feed with hone edge radius $R_{n}=20 \mu \mathrm{m}$ are compared with their corresponding experimental results [10], as shown in Table 1 and Fig.2. A good match of numerical and experimental results can be figured out. For the segmented chip morphology shown in Fig.2(a), $h_{\max }$ and $h_{\min }$ parameters are also highlighted. These parameters are usually used to determine degree of chip segmentation that directly effects machined surface integrity.

During machining process, tool advances in cutting direction (negative x-axis, as per Fig.1 and Fig.2) with certain defined cutting speed and pushes the workpiece material. Material gets elastically and onwards plastically deforms. Sever damage of certain workpiece elements (mainly in front of tool tip) occurs and these elements on acquiring the damage criterion (equation 5) are removed from the mesh. Hence, normal chip formation and its separation process continues. Nevertheless, as tool approaches near workpiece exit edge (along length of workpiece), the unconstrained exit edge (free edge) of workpiece experiences bending load; due to initial elastic flow of workpiece material towards edges (along the length in cutting direction).

As tool further advances, bending load keeps on increasing and a point of maximum stress appears (pivot point) on exit edge. Around this "pivot point" another shear zone develops (termed as "negative shear zone") and enlarges towards tool edge and meets primary shear zone, as shown in Fig.2. Subsequently, stresses in this dual shear zone region (primary and negative shear zones) rises and material in front of tool tip (in chip separation zone) degrades and loses its stiffness. The damage criterion [used in chip separation zone (equation 5)], under these conditions is prematurely met and mesh elements in this region are removed; quite far ahead of tool tip, as shown in Fig.3. Under these conditions, actual 
machining process halts and tool pushes away semi machined chip (boot type chip) and deforms the workpiece exit edge. The shape of deformed edge (as shown in Fig.3) is termed as negative burr with chamfer in literature [2].

Table 1: Cutting forces $(\mathrm{N})$ comparison for a depth of cut $a_{\mathrm{P}}=4 \mathrm{~mm}$ and $\mathrm{R}_{\mathrm{n}}=20 \mu \mathrm{m}$.

\begin{tabular}{lcc}
$\begin{array}{l}\text { Parametric } \\
\text { combinations }\end{array}$ & $\begin{array}{l}\text { Numerical } \\
\text { results }\end{array}$ & $\begin{array}{l}\text { Experimental } \\
\text { results [10] }\end{array}$ \\
\hline $\mathrm{V}_{\mathrm{C}}=800, f=0.3$ & 669 & 769 \\
$\mathrm{~V}_{\mathrm{C}}=400, f=0.3$ & 657 & 764 \\
$\mathrm{~V}_{\mathrm{C}}=800, f=0.4$ & 840 & 976 \\
$\mathrm{~V}_{\mathrm{C}}=400, f=0.4$ & 833 & 971 \\
\hline
\end{tabular}

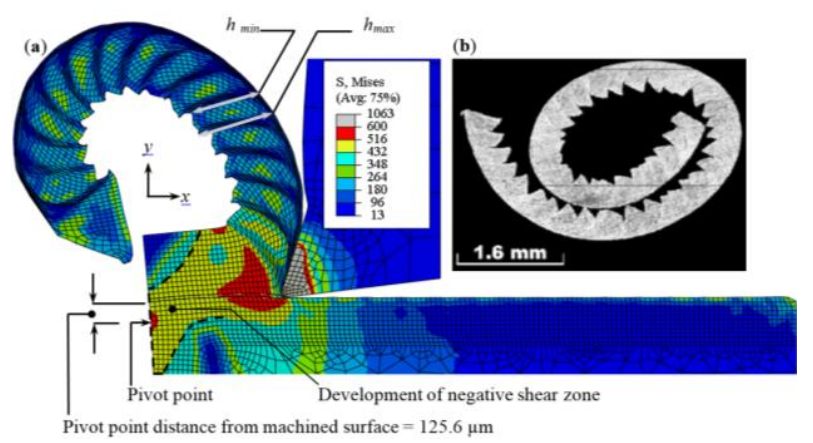

Fig. 2. (a) Numerical and (b) experimental chip [10] comparison for $\mathrm{V}_{\mathrm{C}}=800 \mathrm{~m} / \mathrm{min}$, $f=0.4 \mathrm{~mm} / \mathrm{rev}, \mathrm{R}_{\mathrm{n}}=20 \mu \mathrm{m}$.

In machining of aluminum alloys, two types of exit (end) burr: positive burr (without chamfer) and negative burr (with chamfer) have been witnessed. Cutting parameters and cutting conditions variations can result in either type of burr formation. In the current work, for various combinations of cutting speed, feed and cutting edge only negative burr has been produced for machining of AA2024. Fig.4, shows the burr lengths as function of cutting edges for various combinations of cutting speed and feed. Longer burrs are predicted as cutting edge dimensions (hone edge or chamfer geometry) increases. Similar trends of pivot point location (measured along y-axis from machined surface) have also been figured out. The farther is the location of pivot point from machined surface, longer are the burrs.

Furthermore, to investigate that either thermal softening; promoting flow of material or larger mechanical loadings (for higher tool edge dimensions); causing more widened and deformed plastic zones is the major phenomenon causing bur formation, maximum temperatures and cutting force plots are shown in Fig.5 and 6, respectively.

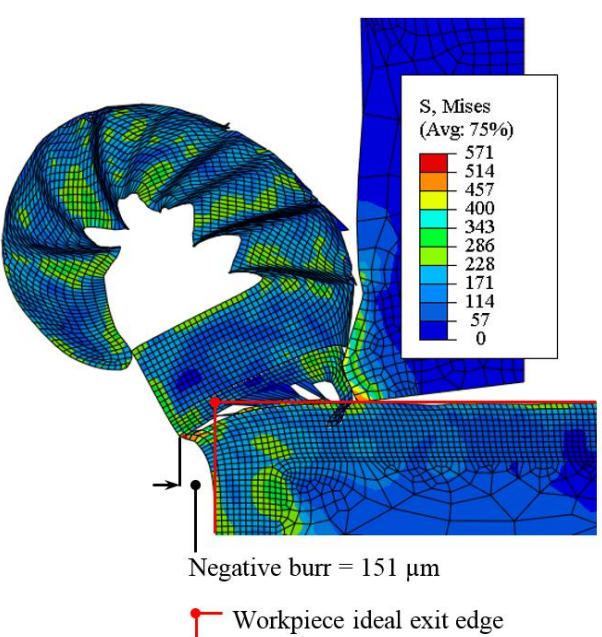

Fig. 3. Boot type chip, material failure in front of tool tip and burr generation with chamfer edge $(\mathrm{req}=180 \mu \mathrm{m}$ corresponding to $1 \beta=0.2 \mathrm{~mm}, \gamma \beta=25^{\circ}$ ) for $\mathrm{VC}=800 \mathrm{~m} / \mathrm{min}, \mathrm{f}=0.4 \mathrm{~mm} / \mathrm{rev}$.

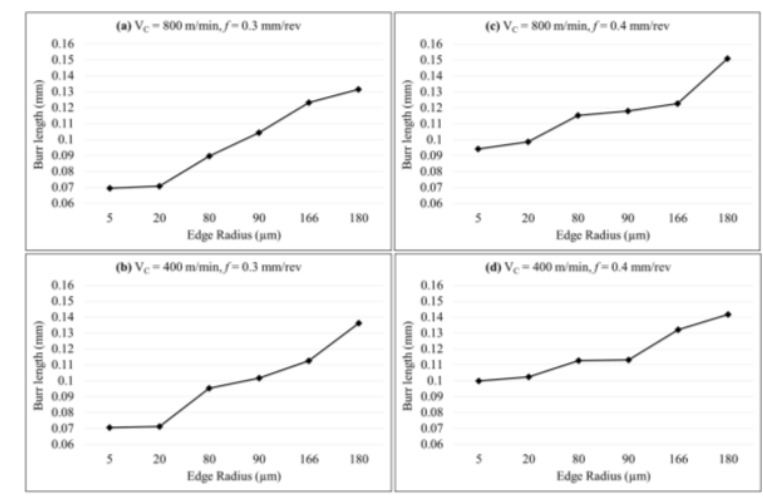

Fig. 4. Burr lengths as function of cutting edge radius.

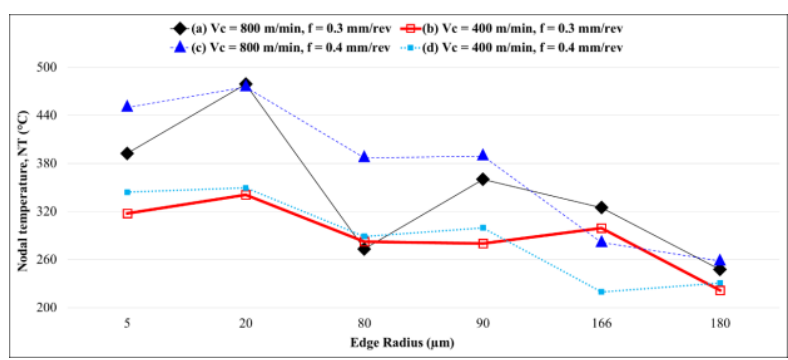

Fig.5. Workpiece maximum temperatures as function of cutting edge radius.

It can be figured out (Fig.5) that as tool edge dimension increases the temperature decreases. Indeed, temperature easily dissipates on larger tool edge surface area. This shows that thermal softening for higher tool edge dimensions is not a dominant phenomenon causing flow of material towards edges and promote burr formation. On the other hand, cutting force plots (Fig. 6) show that, as tool edge dimension increases; higher cutting forces are required to 
deform a wider area experiencing plastic deformation. Now, due to these widened shear zones (primary and negative shear zones) material in front and far ahead of tool tip gets severely deforms and loses its stiffness (Fig.3) and tool pushes away semi-machined chip, while damaging the exit edge (exit burr).

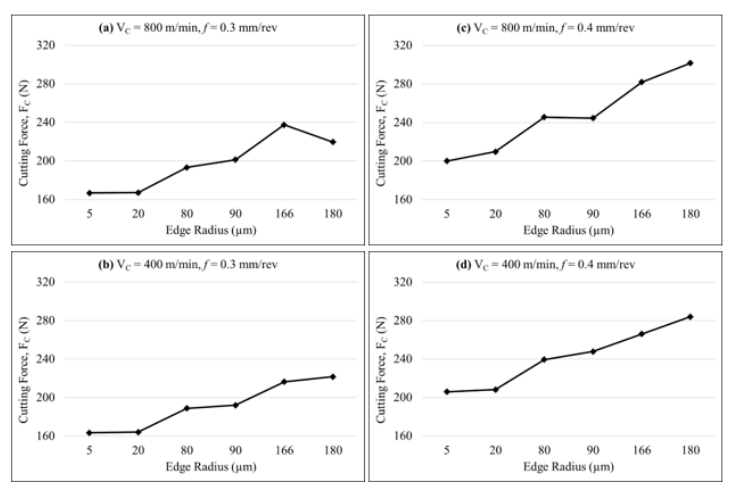

Fig. 6. Cutting forces as function of cutting edge radius.

Finally, chamfer length is the major macroscopic parameter (for chamfer edge tool) which largely influences in widening of shear zone and consequently higher cutting forces are required, as can be figured out by Fig.7 plots. This shows that chamfer length variation can effect burr generation more than chamfer angle variations.

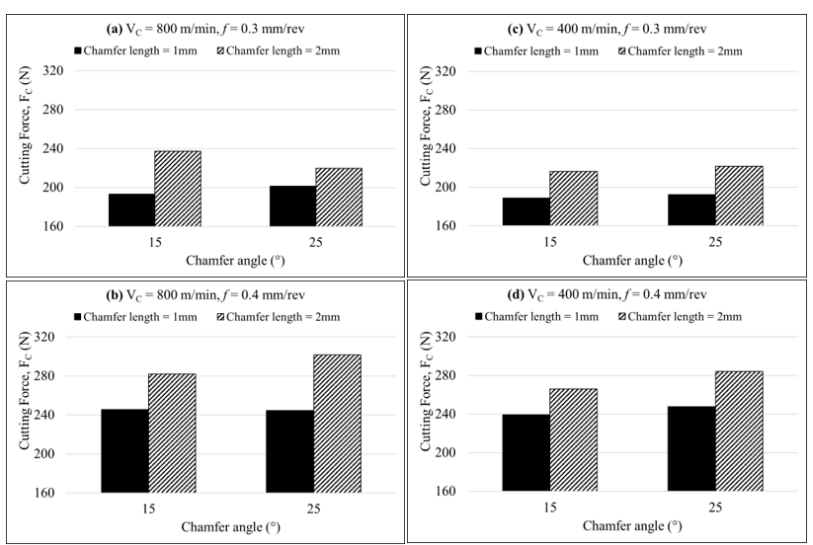

Fig.7. Cutting forces as function of chamfer edge geometry parameters.

\section{Conclusion}

This paper presents a FE based numerical approach on burr formation process in orthogonal cutting of an aluminum alloy AA2024. Objective is to investigate the effects of tool edge geometries (hone and chamfer edge) on exit burr lengths. It has been found that, at higher hone edge radius or chamfer edge dimensions (mainly higher chamfer length) widened and highly stressed shear zones (primary and negative) are produced. These cause premature workpiece material failure in the front and far ahead areas of the tool tip. Actual cutting and shearing process cease and tool pushes away the chip and detached uncut chip material (collectively termed as boot type chip), and plastically deforms workpiece exit edge. Later is termed as negative burr with chamfer. It has been found that as tool edge dimensions increases, longer burr lengths are generated. Machining operation performed with large tool edge geometries causes more widened workpiece area to undergo plastic deformation resulting in higher cutting forces. While, temperatures easily dissipate on large tool area and does not cause significant thermal softening; hence not promoting flow of material towards workpiece edges and burr generation. Furthermore, chamfer length is found to be the dominant parameter (for chamfer edge tool) in widening shear zones and enhancing burr formation. This work aims to provide an insight to machinists and production engineers to optimally select tool edge geometries and cutting parameters to minimize burr formation when dry machining AA2024

\section{References:}

[1] ASM handbook. Machinability, vol. 16. ASM International. 1990.

[2] Régnier T, Fromentin G, et al. Fundamental study of exit burr formation mechanisms during orthogonal cutting of AlSi aluminium alloy. $J$ Mater Process Technol, 257, 2018, 112-122.

[3] Asad M. Effects of Tool Edge Geometry on Chip Segmentation and Exit Burr: A Finite Element Approach. Metals, 11, 2019, 1234.

[4] Aurich J C, Dornfeld D A, Arrazola P J, et al. Burrs-Analysis, control and removal. CIRP Ann: Manuf Tech, 58, 2009, 519-542.

[5] Asad M, Ijaz H, et al. Finite Element Analysis and Statistical Optimization of End-Burr in Turning AA2024. Metals, 9, 2019, 276.

[6] Niknam S A, Songmene V. Statistical investigation on burrs thickness during milling of 6061-T6 aluminium alloy. In Proceedings of the CIRP, 1st International Conference on Virtual Machining Process Technology, Montreal, QC, Canada, 28 May-1 June 2012.

[7] Khanafer K, Eltaggaz A, et al. Toward sustainable micro-drilling of Inconel 718 superalloy using MQL-Nanofluid. Int $J A d v$ Manuf Technol,107, 2020, 3459-3469.

[8] Kim D Y, Kim D M, Park H W. Predictive cutting force model for a cryogenic machining process incorporating the phase transformation 
of Ti-6Al-4V. Int J Adv Manuf Technol, 96, 2018, 1293-1304.

[9] Denkena B, Koehler J, Rehe M. Influnce of the honed cutting edge on tool wear and surface integrity in slot milling of $42 \mathrm{CrMo} 4$ steel. Procedia CIRP, 1, 2012, 190-195.

[10] Mabrouki T, Girardin F, et al. 2008. Numerical and experimental study of dry cutting for an aeronautic aluminium alloy (A2024-T351). Int J Mach Tools Manuf, 481, 2008, 187-197.

[11] Ijaz H, Zain-ul-abdein $\mathrm{M}$ et al. A numerical approach on parametric sensitivity analysis for an aeronautic aluminium alloy turning process. Mechanics, 22, 2016, 149-155.

[12] Saleem W, Asad M, et al. Numerical investigations of optimum turning parametersAA2024-T351 aluminum alloy. Mach Sci Technol, 20, 2016, 634-654.

[13] Ijaz H, Zain-ul-abdein $M$, et al. Numerical simulation of the effects of elastic anisotropy and grain size upon the machining of AA2024. Mach Sci Technol, 22, 2018, 522-542.

[14] Johnson G R, Cook W H. Fracture characteristics of three metals subjected to various strains, strain rates, temperatures and pressures. Eng Fracture Mechanics, 21, 1985, $31-48$.

[15] Ijaz H, Zain-ul-abdein M, et al. Modified johnson-cook plasticity model with damage evolution: application to turning simulation of 2xxx aluminium alloy. J Mechanics, 33, 2017, 777-788.

[16] Ijaz H, Asad M. Prediction of delamination crack growth in carbon/fiber epoxy composite laminates using non-local interface damage model. Mechanics \& Industry, 15, 2014, 293300 .

\section{Nomenclature}

\section{A}

$a_{P}$

(mm)

$B$

$C$

$C_{p}$

$D$

$D_{1} \ldots D_{5}$

E

$f$

$G_{f}$

$K_{C I, I I}$

$m$
Initial yield stress (MPa)

Cutting depth or axial depth of cut

Hardening modulus (MPa)

Strain rate dependency coefficient

Specific heat $\left(\mathrm{Jkg}^{-10} \mathrm{C}^{-1}\right)$

damage evolution parameter

Coefficients of Johnson-Cook material shear failure initiation criterion

Young's modulus (MPa)

Feed rate $(\mathrm{mm} / \mathrm{rev})$

Fracture energy $(\mathrm{N} / \mathrm{m})$

Fracture toughness $(\mathrm{MPa} \sqrt{\mathrm{m}})$ for failure mode $I$ and mode $I I$

Thermal softening coefficient $n$

$P$

$T$

$T_{m}$

$T_{r}$

$\bar{u}$

$\bar{u}_{f}$

$\Delta u$

$V_{C}$

$P / \bar{\sigma}$

$\bar{\varepsilon}$

$\dot{\bar{\varepsilon}}$

$\dot{\bar{\varepsilon}}$

$\bar{\varepsilon}_{f}$

$\Delta \bar{\varepsilon}$

$\bar{\varepsilon}_{0 i}$

$\sigma_{J C}$

$\sigma_{y}$

$\omega$

$v$

\section{Contribution of individual authors to the creation of a scientific article (ghostwriting policy)}

Conceptualization, Muhammad Asad; Formal analysis, Faramarz Djavanroodi and Hassan Ijaz; Methodology, Muhammad Azhar Ali Khan and Muhammad Usman Rashid; Validation, Tarek Mabrouki. Writing - original draft, Muhammad Asad, Muhammad Azhar Ali Khan and Muhammad Usman Rashid; Writing - review \& editing, Faramarz Djavanroodi and Hassan Ijaz.

\section{Sources of funding for research presented in a scientific article or scientific article itself}

Authors acknowledge the financial and technical support provided by Prince Mohammad Bin Fahd University to conduct the research work.

\section{Creative Commons Attribution License 4.0 (Attribution 4.0 International, CC BY 4.0)}

This article is published under the terms of the Creative Commons Attribution License 4.0

https://creativecommons.org/licenses/by/4.0/deed.en_US 